\title{
Correlated biofilm imaging, transport and metabolism measurements via combined nuclear magnetic resonance and confocal microscopy
}

\author{
Jeffrey S McLean ${ }^{1}$, Ositadinma N Ona ${ }^{2}$ and Paul D Majors ${ }^{2}$ \\ ${ }^{1} J$. Craig Venter Institute, La Jolla, CA, USA and ${ }^{2}$ Biological Sciences Division, Pacific Northwest National \\ Laboratory, Richland, WA, USA
}

\begin{abstract}
Bacterial biofilms are complex, three-dimensional communities found nearly everywhere in nature and are also associated with many human diseases. Detailed metabolic information is critical to understand and exploit beneficial biofilms as well as combat antibiotic-resistant, disease-associated forms. However, most current techniques used to measure temporal and spatial metabolite profiles in these delicate structures are invasive or destructive. Here, we describe imaging, transport and metabolite measurement methods and their correlation for live, non-invasive monitoring of biofilm processes. This novel combination of measurements is enabled by the use of an integrated nuclear magnetic resonance (NMR) and confocal laser scanning microscope (CLSM). NMR methods provide macroscopic structure, metabolic pathway and rate data, spatially resolved metabolite concentrations and water diffusion profiles within the biofilm. In particular, current depth-resolved spectroscopy methods are applied to detect metabolites in 140-190 nl volumes within biofilms of the dissimilatory metal-reducing bacterium Shewanella oneidensis strain MR-1 and the oral bacterium implicated in caries disease, Streptococcus mutans strain UA159. The perfused sample chamber also contains a transparent optical window allowing for the collection of complementary fluorescence information using a unique, in-magnet CLSM. In this example, the entire threedimensional biofilm structure was imaged using magnetic resonance imaging. This was then correlated to a fluorescent CLSM image by employing a green fluorescent protein reporter construct of $S$. oneidensis. Non-invasive techniques such as described here, which enable measurements of dynamic metabolic processes, especially in a depth-resolved fashion, are expected to advance our understanding of processes occurring within biofilm communities.
\end{abstract}

The ISME Journal (2008) 2, 121-131; doi:10.1038/ismej.2007.107; published online 22 November 2007

Subject Category: microbial population and community ecology

Keywords: biofilm; diffusion; metabolism; NMR; Shewanella oneidensis; Streptococcus mutans

\section{Introduction}

Bacterial biofilms are communities of cells attached to surfaces and to each other in a matrix of excreted polymeric substances (Stoodley et al., 2002). This metabolically active, heterogeneous structure hinders fluid transport (Stewart, 2003), resulting in concentration gradients for oxygen, $\mathrm{pH}$, nutrients and metabolites. Correspondingly, biofilm bacteria are physiologically and functionally distinct from free-floating bacteria; for example, biofilm cells can

Correspondence: JS McLean, Environmental microbial genomics, J. Craig Venter Institute, 11149 N Torrey Pines Rd, suite 220, La Jolla, CA 92037, USA.

E-mail: jmclean@jcvi.org

Received 7 August 2007; revised 21 October 2007; accepted 24

October 2007; published online 22 November 2007 be highly resistant to antibiotics (Costerton et al., 1999; Stewart, 2001; Davies, 2003) potentially due to slowed or reduced metabolism in substrate-limited areas within the structure (Stewart, 2003). As a result, treating biofilm diseases and persistent infections (Costerton et al., 1999) such as on medical implants are a significant challenge.

Detailed metabolic information on the microscale is critical to understand and exploit beneficial biofilms and combat antibiotic-resistant, diseaseassociated biofilm-forming species. These temporally and spatially variable metabolic gradients that occur at the micrometer scale are extremely difficult to measure and gain access to with current technology. Microelectrodes offer a way to map a single chosen parameter, for example, $\mathrm{pH}$ or $\mathrm{pO}_{2}$ (Revsbech, 2005); however, the probes physically perforate the sample thereby changing its 
permeability and therefore potentially its metabolism. Confocal laser scanning microscopy (CLSM) is widely used for biofilm investigations because of its non-invasive nature and its three-dimensional (3D) resolution capability, although optical penetration depth is limited due to optical absorption and scattering phenomena (Vroom et al., 1999). Twophoton CLSM methods overcome depth penetration concerns however; both two-photon and CLSM typically require the addition of fluorescent tracers when detecting metabolic activity that could have undesirable effects on the cellular function (Parolin et al., 1990; Ullrich et al., 1996). Fluorescence in situ hybridization and microautoradiography provide species and substrate-uptake information at the single-cell level, but are destructive and assay only one substrate per sample. Thus, few techniques exist that can continuously measure biofilm metabolite profiles in a truly non-invasive and non-destructive manner with adequate time and spatial resolution.

Nuclear magnetic resonance (NMR) is a noninvasive method that provides a non-invasive, subatomic view of molecular chemical, physical and transport processes. NMR is non-destructive, non-sample consuming and is insensitive to sample opacity. NMR spectroscopy techniques provide detailed metabolic information by analysis of cell extracts, supernatants, biological fluids and live biological samples. ${ }^{1} \mathrm{H}$ and ${ }^{1} \mathrm{H}$-detected ${ }^{13} \mathrm{C}$ NMR provide direct, time-resolved monitoring of metabolite concentrations. The disadvantage of NMR is its inherent low sensitivity due to the low energies involved, requiring careful optimization to reduce measurement times and lower concentration detection thresholds. NMR has been used to measure flow and diffusion in biofilm systems (Lewandowski, 1992 \#198; Lewandowski, 1993 \#197) (Lewandowski et al., 1995; Vogt et al., 2000; Van As and Lens, 2001; Seymour et al., 2004) as well as planktonic cell metabolism (Shanks, 2001). Previous NMR studies of live prokaryotic cell suspensions, gelimmobilized cells and extracts have provided detailed metabolic information (Junter et al., 2002). Several NMR studies have explored extracts or concentrated samples of cells grown as biofilms (Vogt et al., 2000; Mayer et al., 2001; Hu et al., 2004).

Recently, we employed a combined CLSM and NMR microscope (Wind et al., 2000) to measure time (Majors et al., 2005a) and, also for the first time, depth-resolved metabolite concentrations under flow in live biofilms (Majors et al., 2005b). Unlike the traditional flow-tube geometry used for biofilm imaging and diffusion studies, this application employs a growth media perfusable sample chamber of planar geometry (Figure 1a and Materials and methods) that supports a sample grown onto a thin surface (typically a no. 1 glass coverslip). Using this system, we can now apply a series of combined techniques to perform magnetic resonance imaging (MRI), magnetic resonance spectroscopy (MRS) and also diffusion in a novel way on a single biofilm sample. We describe and demonstrate these techniques using two model systems: (1) Shewanella oneidensis strain MR-1, a metal-reducing, environmentally important strain (Myers and Nealson, 1988; Heidelberg et al., 2002) studied for its bioremediation and bioenergy potential and (2) Streptococcus mutans strain UA159 (Ajdic et al., 2002), an oral bacterium implicated in oral caries disease (Poole and Newman, 1971; Marsh, 2003).

\section{Materials and methods}

\section{Biofilm cultivation}

Controlled biofilm samples of defined geometry were pregrown in a homebuilt biofilm fermenter based on the constant depth film fermenter design (CDFF) (Dibdin and Wimpenny, 1999). A CDFF enables the generation of multiple biofilm samples of defined depths of $100-500 \mu \mathrm{m}$ on a $5 \mathrm{~mm}$ diameter substratum before transferring to the NMR. The homebuilt system consisted of a $500 \mathrm{ml}$ Sixfors bioreactor vessel (Infors AG, Bottmingen, Switzerland), which was modified in-house to function as a CDFF, in order to generate steady-state biofilms (Wimpenny, 1985). The modification consists of a PEEK plastic disc-shaped plate (stationary) insert with six sample wells that support $5 \mathrm{~mm}$ glass coverslips or other substratum and a (rotating) Teflon scraper blade attached to the vertical magnetic stirrer shaft that shears the plate surface to remove excess biomass.

\section{Shewanella oneidensis biofilms}

The organism S. oneidensis MR-1 was used in this study with a transcriptional reporter fusion. The plasmid p519ngfp (ATCC 87453; Matthysse et al., 1996) contains an npt-2 promoter in front of green fluorescent protein and was inserted in MR-1 as described in Gonzalez-Gil et al. (2005). A modified formulation of chemically defined medium M1 (Zachara et al., 1998) was used for growth and metabolism studies. The base components of the medium consisted of $\left(\mathrm{g}^{-1}\right)$ PIPES (0.907) buffer, $\mathrm{NaOH}$ (0.3), $\mathrm{NH}_{4} \mathrm{Cl}$ (1.5), KCl (0.1), $\mathrm{NaH}_{2} \mathrm{PO}_{4}$ (0.6), $\mathrm{Na}_{2} \mathrm{SO}_{4}(0.213)$ and $\mathrm{CaCl}_{2}$ (0.1) with additions of vitamins, minerals and amino acids. D,L-Lactate $(18 \mathrm{mM})$ and fumaric acid $(35 \mathrm{mM})$ were added for all experiments. Additionally, the culture medium was amended with $50 \mu \mathrm{g} \mathrm{ml}^{-1}$ kanamycin during pregrowth in the continuous bioreactor (see below) and in the NMR experiments in order to ensure the retention of plasmids bearing the fluorescent proteins. For Shewanella biofilms, cells were inoculated onto $5 \mathrm{~mm}$ no. 1 glass coverslips in the CDFF from an overnight culture in trypticase soy broth and were allowed to sit for $15 \mathrm{~min}$ before a feed of sterile modified M1 media was supplied at $0.5 \mathrm{ml} \mathrm{min}^{-1}$. The temperature was maintained at $30{ }^{\circ} \mathrm{C}$ in the CDFF. 

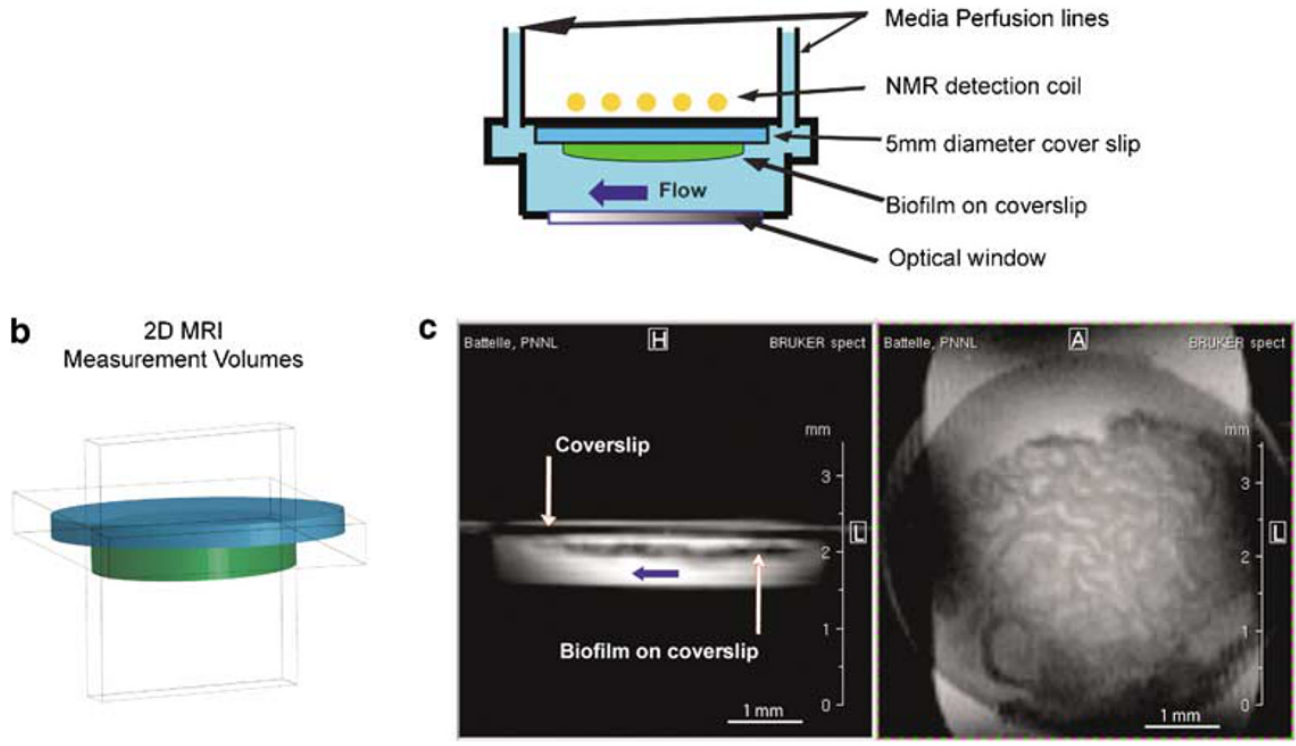

d

e f
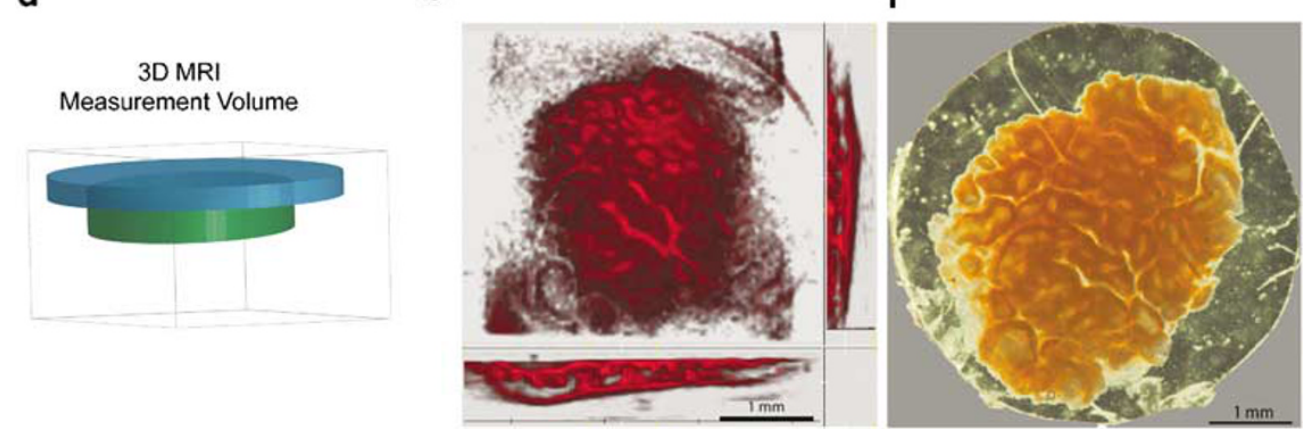

Figure 1 Representative 2D and 3D MRI for a Shewanella oneidensis (strain MR-1) biofilm. (a) Schematic of the planar NMR chamber (b) Illustration of the coverslip-attached biofilm showing the locations of the 2D MRI measurement volumes. (c) Water-selective 2D MRI for a Shewanella biofilm (side and top views). Blue arrow indicates the direction of flow through the chamber. Note: The scale bar is oriented vertically in the 2D MRI images. (d) Illustration showing the location of the 3D MRI measurement volume. (e) Multiple-slice rendering of a 3D MRI for the biofilm in (c) showing three orthogonal views. (f) Transmitted light image of the same biofilm after removal from the sample chamber, demonstrating the accurate capture of macroscopic structure by MRI.

S. mutans biofilms

S. mutans strain UA159 (kindly provided by Dr Wenyuan Shi, UCLA School of Dentistry) was maintained on brain-heart infusion (BHI, Difco, Detroit, MI, USA) broth. For cultivation of S. mutans biofilms in the CDFF, an overnight culture grown in $\mathrm{BHI}$ at $37^{\circ} \mathrm{C}$ was diluted 10 -fold in 1/10th strength $\mathrm{BHI}$ and used to inoculate the CDFF. Biofilms were pregrown for 1 week on 5-mm-diameter coverslips or hydroxyapatite disc with the media feed rate set at $0.2 \mathrm{ml} \mathrm{min}^{-1}$. The temperature was maintained at $37^{\circ} \mathrm{C}$. The CDFF biofilm growth media for $S$. mutans consisted of $1 / 10$ th strength BHI with $8 \mathrm{gl}^{-1}$ sucrose and $0.1 \mathrm{~g} \mathrm{l}^{-1} \mathrm{CaCl}_{2}$. For studies conducted with sucrose on hydroxyapaptite discs (Clarkson Chromatography Inc., PA, USA), a rich undefined formulation (BHI $+8 \mathrm{gl}^{-1}$ sucrose) was used.

For the glucose metabolism studies, a separate perfusion medium designed to be NMR compatible and provide a single carbon source was used. This chemically defined medium was composed of following (in grams per liter/millimolar concentration): $\left(\mathrm{NH}_{4}\right)_{2} \mathrm{SO}_{4}, 0.8 / 6.0 ; \mathrm{NaCl}, 0.6 / 10.0$; ascorbic acid, 0.5/2.5; glucose, 2.5/13.9; $\mathrm{MgCl}_{2} \cdot 6 \mathrm{H}_{2} \mathrm{O} 0.16 /$ 0.78; $\mathrm{CaCl}_{2} \cdot 2 \mathrm{H}_{2} \mathrm{O}, \quad 0.01 / 0.07 ; \mathrm{KH}_{2} \mathrm{PO}_{4}, 3.0 / 22.0$; $\mathrm{K}_{2} \mathrm{HPO}_{4}$, 2.5/14.0; cysteine hydrochloride, 0.3/1.7. To this, $10 \mathrm{ml}^{-1}$ of a vitamin mix was added. The composition of the vitamin-mix (in $\mathrm{gl}^{-1}$ ) was biotin, 0.002; folic acid, 0.002; pyridoxine hydrochloride, 0.01; riboflavin, 0.005; thiamine, 0.005; nicotinic acid, 0.005; pantothenic acid, 0.005; vitamin B-12, 0.0001; p-aminobenzoic acid, 0.005 and thioctic acid, 0.005 . Glucose utilization stop-flow experiments were performed with $2 \mathrm{~mm}$ phosphate buffer $\mathrm{pH} 7$ (physiologically relevant concentration) and without buffer.

For the $S$. mutans glucose studies, NMR spectroscopy was used to monitor the absolute concentrations of the metabolites produced by the biofilms under laminar flow rates and after flow was halted. Initially, the flow was started at $12 \mathrm{ml} \mathrm{h}^{-1}$ after addition of each new medium formulation (buffered and unbuffered) and was allowed to reach a 
stable concentration $(\sim 20-25 \mathrm{~min}=160-200$ volume changes) after which the flow was stopped and a 'closed-batch' system was created. Bulk spectroscopy measurements (described below) for this stop-flow kinetic study were performed prior to and after the flow was halted. Additional measurements on replicate $S$. mutans CDFF biofilm samples were conducted by repeating the stop-flow kinetic experiment in a $\mathrm{pH}$ flow-through cell (Cole-Parmer $800 \mu \mathrm{l}$ volume, $3 \mathrm{~mm}$ ID) fitted with PEEK tubing and maintained at $37^{\circ} \mathrm{C}$ in a water bath.

\section{NMR chamber and flow system}

The nuclear magnetic resonance/optical microscope employs a Bruker Instruments (Billerica, MA, USA) Avance digital NMR spectrometer running Bruker TopSpin/Paravision version 4.0 imaging software, with homebuilt top-loading magnetic field gradient and bottom-loading optical inserts installed in its $89 \mathrm{~mm}$ vertical bore superconducting magnet (Majors et al., 2005a). Biofilm studies were performed using a perfusable NMR flow chamber designed to support one biofilm sample attached to a $5 \mathrm{~mm}$ circular coverslip as described previously (Minard et al., 2004). The sample chamber was installed in the test section of a $11.7 \mathrm{~T}$ superconducting $89 \mathrm{~mm}$ bore magnet corresponding with a Larmor precession of $500.44 \mathrm{MHz}$ for hydrogen-1. Measurements include 2D and 3D MRI of biomass volume and distribution, MRS and spectroscopic imaging (MRSI) of supernatant metabolite concentrations and water diffusion imaging.

The planar NMR microscopy sample chamber (Figure 1a) consists of a face-sealing sample chamber integrated with an NMR detection coil on top and an optical (CLSM) window at the bottom. The perfusion lines are attached to an NMR magnet insert containing a radiofrequency detection circuit (Minard et al., 2004). When assembled, the 5-mmdiameter coverslip sample is supported $250 \mu \mathrm{m}$ below the planar (RF) NMR microcoil and $900 \mu \mathrm{m}$ above the transparent optical window with the biofilm oriented toward the optical window. The biofilm faces downward into a rectangular, $4 \mathrm{~mm}$ wide $\times 1.17 \mathrm{~mm}$ deep flow channel, which results in a total liquid volume of $25 \mu \mathrm{l}$. The perfusion system was set up as described in Majors et al. (2005a). Perfusion lines inject and remove growth media at opposite ends of and in a direction normal to the coverslip. The flow rate was controlled in these experiments using a pulseless dual syringe pump (Pharmacia P-500, Uppsala, Sweden) and the sample temperature was maintained at $30 \pm 1{ }^{\circ} \mathrm{C}$ for $S$. oneidensis studies and $37 \pm 1{ }^{\circ} \mathrm{C}$ for $S$. mutans studies by purging the magnet bore with $\mathrm{N}_{2}$ gas using a controlled temperature gas stream delivery unit (FTS Systems, Stone Ridge, NY, USA). Oxygen partial pressure was not monitored or controlled in these experiments although this and previous studies (Majors et al., 2005b) have shown that anaerobic respiration is observed, which indicates oxygen is limited. Flow over the biofilm region was calculated and measured to be laminar for flow rates from 1 to $12 \mathrm{ml} \mathrm{h}^{-1}$ (corresponding with 0.66-7.9 $\mathrm{min}^{-1}$ dilution rates) (PD Majors, unpublished data) employed in this study.

\section{Magnetic resonance imaging}

Two- and three-dimensional MRI techniques are routinely employed to visualize the biofilm samples. Fast (2-10 min), multidirectional slice-selective two-dimensional (2D) MRI yields three mutually perpendicular slices. For 3D microscopic MRI imaging, the entire volume was measured and rendered as a series of contiguous voxels comprising the volume around the sample, with a single intensity for each voxel representing its NMR signal. The detection coil sensitivity is designed for best performance over the coverslip center and leads to artificially diminished intensity near the chamber boundaries.

\section{Confocal microscopy}

For the combined NMR and CLSM study, $S$. oneidensis strain MR-1 with a constitutive reporter fusion for green fluorescent protein expression (p519nGFP) (Gonzalez-Gil et al., 2005) was grown in the CDFF with lactate and fumarate in M1 medium. Fluorescent microscopic observations were performed on the integrated NMR CLSM described in Minard et al. (2004). Briefly, the components of the CLSM include a compact solid-state laser that is operated at $488 \mathrm{~nm}$ (Coherent Inc., Santa Clara, CA, USA), and two cooled photomultiplier tubes (H7422P-40; Hamamatsu Corp., Bridgewater, NJ, USA) to detect red and green fluorescence. A non-metallic objective (Special Optics, Wharton, NJ, USA) was used with a numerical aperture of 0.5 resulting in a resolution of $0.7 \mu \mathrm{m}$ in a horizontal plane and $10 \mu \mathrm{m}$ in the vertical direction. During the biofilm studies, we acquired optical images with a planar resolution of $1-2 \mu \mathrm{m}$ over an area of $2490 \times 2490 \mu \mathrm{m}^{2}$ using a laser excitation power on the order of $0.1 \mathrm{~mW}$ (at the sample). A total of 61 sections at a step size of $5 \mu \mathrm{m}$ were employed to generate the confocal images. Three-dimensional images were created using the software Volocity (Improvision Ltd, Lexington, MA, USA) and the top-down views with side profile panels showing biofilm depth using IMARIS (Bitplane AG, Zürich, Switzerland).

\section{Magnetic resonance spectroscopy}

The NMR measurements included localized spectroscopy (Majors et al., 2005a) to measure the average concentrations of hydrogen-containing compounds. The localization procedure avoids magnetic distortions (and associated spectral broadening) located 
near the edge of the glass coverslip by selecting a columnar volume centered on the slip. These localized MRS data are processed by applying $5 \mathrm{~Hz}$ of exponential line broadening (noise reduction) followed by Fourier analysis to yield spectra, and included an algorithm to suppress resonances within $0.125-0.625$ p.p.m. of the water resonance (the ${ }^{1} \mathrm{H}$ NMR resonance for water is temperature dependent and varies between 4.62 and 4.85 p.p.m. for these studies $\left(T=37\right.$ and $30^{\circ} \mathrm{C}$, resp.)). The resulting spectra were adjusted to yield all positive intensities with first-order phase correction and the baseline was corrected using a multiple (20-30) point splinefitting routine. The data are processed into spectra, the individual resonances integrated and the resultant spectral areas used to calculate the metabolite concentrations. A typical repetition time was $4 \mathrm{~s}$, and the echo time was 5.25-8 ms. Calibration was performed by collecting non-water-suppressed MRS and calibrating the water concentration to $55 \mathrm{M}$. Additionally, Shewanella studies contained $3 \mathrm{~mm}$ PIPES buffer, which was used to provide an internal concentration reference. Finally, the different metabolites were corrected for their respective proton spin counts to obtain water, buffer, substrate and by-product molar concentrations. NMR sensitivity (signal to noise ratio per unit time) performance allows for the measurement of $1 \mathrm{~mm}$ metabolite concentrations in approximately $10 \mathrm{~min}$.

\section{Depth-resolved metabolism and transport measurements}

Depth-resolved metabolite measurements employed a modified MRSI method (Majors et al., 2005b). Sixty-four, $2 \mathrm{~s}$ averages were collected with an echo time of $8 \mathrm{~ms}(\mathrm{TR} / \mathrm{TE}=2000 / 8 \mathrm{~ms})$ for each of 64 phase-encoding steps over a $2 \mathrm{~mm}$ field of view, yielding a depth resolution of $31.2 \mu \mathrm{m}$ in $2.25 \mathrm{~h}$. Data were processed by 2D Fourier transformation. Depth-resolved apparent diffusion measurements employed a standard 1D pulsed magnetic field gradient spin-echo imaging experiment (Callaghan, 1984) (Bruker m_diffse; TR/TE $=4000 / 8.15 \mathrm{~ms}$ ) with a $2.5 \mathrm{~mm}$ slice thickness and readout gradient aligned normal to the coverslip and covering a FOV of $2 \mathrm{~mm}$ with 128 measurements for a depth resolution of $15.6 \mu \mathrm{m}$. Two identical, rectangular gradient pulses (oriented $45^{\circ}$ from the direction normal to the coverslip; gradient pulse duration $\delta=1.2 \mathrm{~ms}$ and center-to-center gradient pulse separation $\Delta=4 \mathrm{~ms}$ ) were symmetrically displaced around the refocusing RF pulse. Ten experiments were performed each with 16 repetitions for a total measurement time of $10.7 \mathrm{~min}$, with differing diffusion weighting factor per experiment (Lebihan, 1990) $(b=285,548,839,988,1527,2065,2475$, 3045 and $3679 \mathrm{~s} \mathrm{~mm}^{-2}$ ). The resulting profile intensities $S$ were fit-point by point-to a first-order diffusion expression $S=\exp (-b D)$ to obtain the depth-resolved quantitative diffusion rate profile.
S. oneidensis biofilms were perfused with M1 media containing lactate and fumarate in the NMR chamber under laminar flow conditions for the correlated imaging, depth-resolved metabolism and diffusion experiments. S. mutans depth-resolved metabolite studies on hydroxyapatite were performed during flow of BHI containing $8 \mathrm{~g} \mathrm{l}^{-1}$ sucrose.

\section{Results}

Magnetic resonance imaging of biofilm structure

A cross-section of the planar NMR microscopy sample chamber with the biofilm facing downward is detailed in Figure 1a. Glass and hydroxyapatite (a model tooth surface) were determined to be acceptable biofilm-support materials that did not cause significant broadening of the ${ }^{1} \mathrm{H}$ NMR spectral line widths for liquids in close proximity to their surface.

Two MRI techniques, 2D and 3D MRI, are routinely employed to visualize the samples and are compared in Figure 1. Fast (2-10 min), multidirectional slice-selective 2D MRI yields three mutually perpendicular slices (two of the three slices comprising the 2D MRI measurement volume are illustrated in Figure 1b). This relatively rapid imaging technique produces an estimate of biofilm mass and architecture, and allows careful alignment of the flow direction with the imaging gradient directions and planning of optimal location and size for the spectroscopy measurement volume (cf. Figure 3a). Figure 1c shows side and top-down images for a $S$. oneidensis biofilm where the intensity or brightness corresponds primarily with the volume fraction of water in that location (waterselective imaging). The areas of reduced intensity within the sample chamber center correspond with the circular glass coverslip and dense biofilm.

Microscopic spin-echo 3D MRI measurements provide better details of the biomass structure and distribution and also enable quantification of biofilm parameters such as biovolume and surface area. Three-dimensional MRI measurement volume is illustrated in Figure 1d. Three-dimensional MRI contrast (Figure 1e) is intentionally biofilm selective; that is, the MRI signal is spin-lattice $\left(\mathrm{T}_{1}\right)$ relaxation weighted to emphasize intra- and extracellular water in close association with the biofilm. Unlike optical methods, ${ }^{1} \mathrm{H}$ MRI has effectively no depth limitation and is used to non-invasively monitor biofilm internal structure, biovolume and distribution over time (Supplementary Video 1). However, 3D MRI is a somewhat time-consuming experiment requiring up to $4.5 \mathrm{~h}$ to obtain images with $60 \times 60 \times 20 \mu \mathrm{m}^{3}$ spatial resolution (highest resolution in the biofilm depth direction) (Majors et al., 2005a). A transmitted light stereoscope image is shown in Figure $1 \mathrm{f}$ for the same biofilm after removal from the chamber, demonstrating close correlation of MRI with optically visible structure. 
Combined MRI and CLSM imaging

A homebuilt CLSM magnet insert (Minard et al., 2004) is used to image the biofilm without moving the sample. Three-dimensional, dual-channel images are collected as a stack of depth-resolved 2D planes, typically requiring several minutes to acquire and are primarily rate limited by the objective translation time. The confocal laser scanning microscope component provides the opportunity to capture and correlate complementary optically detected information. Figure 2 compares consecutively acquired 3D MRI and CLSM rendered images for an MR-1 biofilm containing a green fluorescent protein reporter for a constitutively expressed gene (Gonzalez-Gil et al., 2005). The images can be precisely registered prior to analysis using a dual fluorescent and an MRI detectable

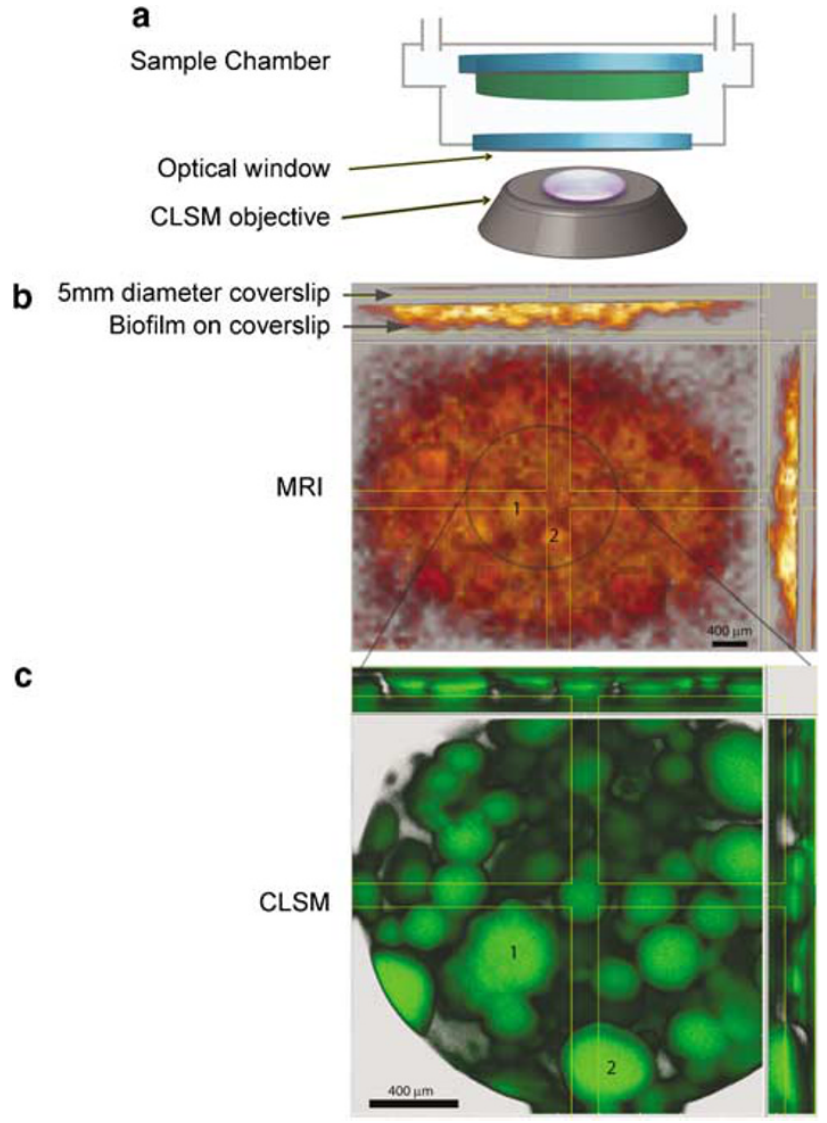

Figure 2 Combined NMR/CSLM sample configuration and representative 3D NMR and CSLM images for a $S$. oneidensis biofilm. (a) Illustration of the perfused NMR sample chamber containing an ex situ-grown biofim attached to the bottom of a $5 \mathrm{~mm}$ glass coverslip. (b) Biofilm-selective 3D MRI revealing full depth penetration without loss of signal intensity. Arrows indicate positions of the $\sim 150 \mu \mathrm{m}$ coverslip and the biofilm. Top panels of the biofilm surface and side panels image planes show internal structure and biomass depth. (c) In-magnet 3D CLSM top down and side views of the same biofilm (spatial registration is approximate). Non-invasive fluorescent imaging was enabled by a green fluorescent protein (GFP) construct in MR1. Yellow cross-hair indicate the location and width of the image planes used to generate the side views. Colonies 1 and 2 are identified in both MRI and CLSM images. fiduciary marker (data not shown). Whereas MRI provides a low-resolution image of the entire sample chamber and internal structure of the biofilm with no opacity or scattering losses, CLSM provides a higher-resolution image within a $1.25 \mathrm{~mm}$ diameter circular FOV centered on the coverslip. The current objective used in this system, however, does not provide sufficient magnification for single cells to be resolved at this time. Importantly, MRI in contrast to fluorescence microscopy does not require altering the sample by using reporters or addition of stains that could have undesired effects on metabolism and viability.

\section{Localized spectroscopy for metabolite profiles}

The capability of NMR to quantify multiple metabolites in biofilms in near real time is the most powerful and unique of the techniques employed. In particular, we use water-suppressed proton MRS localized to an $\sim 6 \mu \mathrm{l}$ rectangular volume (Figure 3a) centered on the $5 \mathrm{~mm}$ diameter coverslip and including the entire chamber height (Majors et al., 2005a). This yields the concentrations of covalent hydrogen-containing metabolites averaged over the sample (biofilm and supernatant fluid). These measurements are non-contacting, non-destructive and non-sample-consuming; thus, they can be repeated to obtain time-resolved metabolite profiles under controlled growth conditions including a completely anaerobic environment. Analogous determinations by traditional techniques such as liquid chromatography or mass spectroscopy would require the destruction of multiple parallel samples and introduce sample variance issues. Figure $3 \mathrm{~b}$ shows MRS data collected for a $S$. mutans strain UA159 (Ajdic et al., 2002) biofilm. For these studies, chemically defined medium containing $14 \mathrm{mM}$ glucose as a single carbon source both with and without buffer was used. A stop-flow kinetic study was performed by perfusing the media at $12 \mathrm{ml} \mathrm{h}^{-1}$ and allowed to reach a stable concentration $(\sim 20-$ $25 \mathrm{~min}=160-200$ volume changes) then halting the inflow of media and following metabolism via timeresolved MRS (8.5 min averages). The glucose fermentation products were lactate and acetate for both conditions. The glucose consumption rate was $8.05 \mathrm{mMh}^{-1}$ in the buffered medium and was reduced to $1.95 \mathrm{mM} \mathrm{h}^{-1}$ in the unbuffered medium (Figure 3c). Parallel experiments using a flowthrough $\mathrm{pH}$ probe (Figure $3 \mathrm{~d}$ ) revealed that the $\mathrm{pH}$ remained relatively stable throughout the experiment when buffer was present and dropped below the critical enamel demineralization value ( $\mathrm{pH}$ 5.5) after $2 \mathrm{~h}$ for the unbuffered system. These results indicate that $S$. mutans tolerate acidic conditions to utilize glucose, albeit at a reduced rate. In addition, corresponding 3D MRI experiments established that the biofilm structure and volume (calculated at $5.5 \mathrm{~mm}^{3}$ ) did not change during acid production (Figure 3e). 

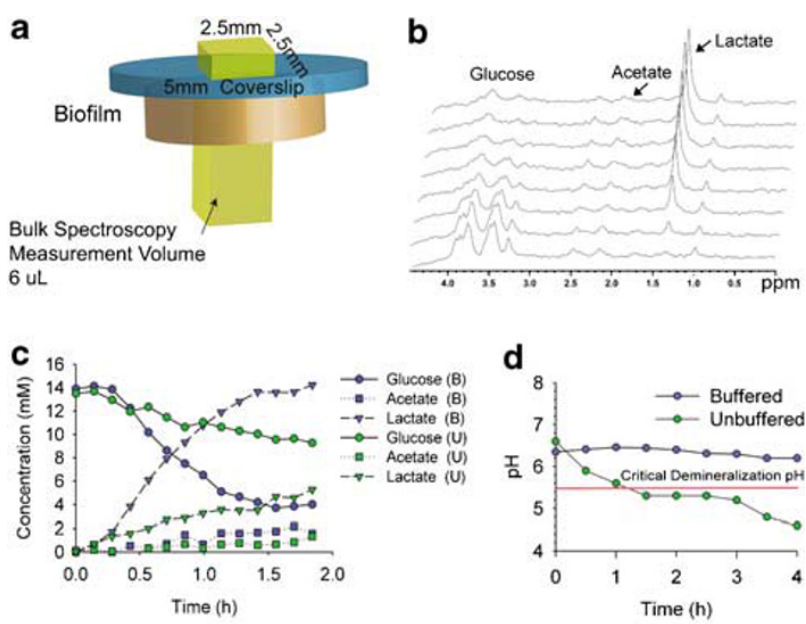

e

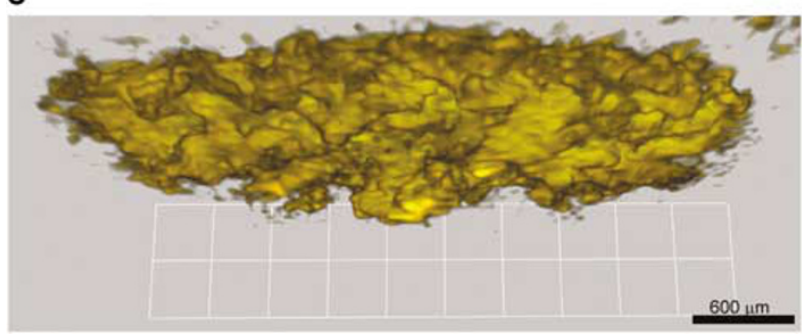

Figure 3 Representative bulk metabolism experiment for an oral (S. mutans) monoculture biofilm in chemically defined media (CDM). The biofilm was pregrown in a constant depth film fermentor to a depth of $400 \mu \mathrm{m}$ and aseptically transferred to the NMR chamber and perfused with fresh buffered CDM with glucose. (a) Illustration showing the rectangular measurement volume and location (yellow box), which includes both biofilm and adjacent supernatant fluid in the chamber. (b) Time-resolved stack plot of NMR spectra during glucose metabolism showing utilization of glucose (4.0-3.2 p.p.m.) and production of acetate (1.9 p.p.m.) and lactate (1.3 p.p.m.) over time. (c) Time-resolved concentrations for a stop-flow kinetics (flow followed by shut-in) of glucose metabolism captured using bulk spectroscopy techniques in a buffered (blue) and unbuffered (green) CDM. (d) Corresponding $\mathrm{pH}$ profiles for each medium type in panel $\mathrm{c}$ conducted in parallel using a perfusable pH chamber. (e) 3D MRI biofilm-selective image of the biofilm (bottom up view).

Correlating depth-resolved spectroscopy and diffusion Magnetic resonance spectroscopy is readily hybridized with MRI to yield MRSI (Brown et al., 1982), which provides spatially resolved spectral (metabolic) data. Specifically, an MRI phase-encoding gradient is applied normal to the biofilm depth to spatially resolve the bulk $6 \mu \mathrm{l}$ MRS measurement volume as contiguous thin volumes (see schematic in Figure 4a)-typically $22-31.2 \mu \mathrm{m}$ thick and corresponding to $140-195 \mathrm{nl}$ volumes. Their corresponding volumes are equivalent to isotropic voxels of 520 and $580 \mu \mathrm{m}$ on a side, respectively. Average local metabolite concentrations as low as $1 \mathrm{~mm}$ are measured and typically require $30 \mathrm{~min}-2 \mathrm{~h}$. These voxel volumes and concentrations correspond with approximately $10^{14}$ metabolite molecules, and closely approximate the number of molecules per voxel for our system when performing microscopic
MRI of water $(55 \mathrm{M})$ at the best spatial resolution of $15 \mu \mathrm{m}$. Thus, in-plane resolution is sacrificed to improve biofilm depth resolution and is allowable due to the planar geometry of the sample imposed by the support surface. Although these measurements can potentially be as thin as a few microns, measurement time, adequate metabolite concentrations and NMR considerations, such as available gradient strengths and diffusive signal losses, dictate the minimum volume attainable to get a spectral signature for metabolites of interest.

A first demonstration of correlated MRI, depthresolved metabolism and depth-resolved water diffusion measurements for a $S$. oneidensis biofilm obtained under flowing conditions is described in Figure 4. MRI was used to quantify and spatially locate the biomass in the chamber (Figures $4 \mathrm{~b}$ and $\mathrm{c}$ ) for subsequent height correlation with MRSI and diffusion measurements. A localized MRSI containing depth-resolved spectral (metabolic) data is shown in Figure 4d. In this representation, the vertical direction corresponds with spatial depth (31.2- $\mu \mathrm{m}$ depth resolution) and the horizontal direction is the ${ }^{1} \mathrm{H}$ NMR spectral dimension, resolving metabolites by their spectral signatures. The colors represent the relative spectral intensities and are related to the chemical concentrations. Figure 4e shows the corresponding depth-resolved water diffusion measurements obtained via 1D diffusionweighted MRI (Callaghan, 1984). This 11 min measurement procedure provides quantitative, depthresolved apparent water diffusion rate profiles $\left(D_{\text {app }}\right.$, the measured local diffusion rate, which includes the bounding effects of the biofilm matrix) that are spatially registered with the MRSI data. The spectral lines for lactate (electron donor), acetate (lactate oxidation product generated in the biomass), fumarate (electron acceptor) and succinate (fumarate reduction product generated in the biomass) are resolved (Figure 4d). Lactate is supplied continuously and reaches a steady-state concentration profile that is depleted at a particular depth. This depth corresponds closely with the biofilm-bulk fluid boundary revealed visually by MRI (Figure 4c) and is also located in the region where the apparent diffusion rate decreases (Figure 4e). Lactate, the electron donor in this case, is therefore rapidly oxidized by the cells and becomes limited in the biomass.

Depth-resolved spectral measurements are critical to quantify where metabolites accumulate for both fundamental and practical applications such as microbially influenced corrosion, host-pathogen interactions and tooth enamel demineralization by oral bacteria. Importantly, biofilms growing within areas that cannot be physically sampled intact could be imaged and monitored using the penetrating nature of NMR. For example, a $S$. mutans biofilm grown on a model tooth surface (hydroxyapatite disc) within a depth-modified NMR chamber (Figure 5) was used as a simple model to more closely 


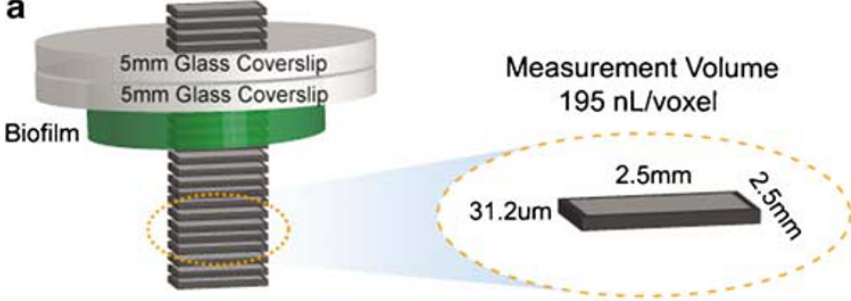

b

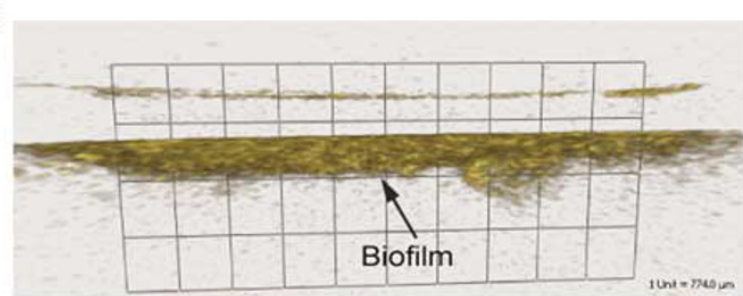

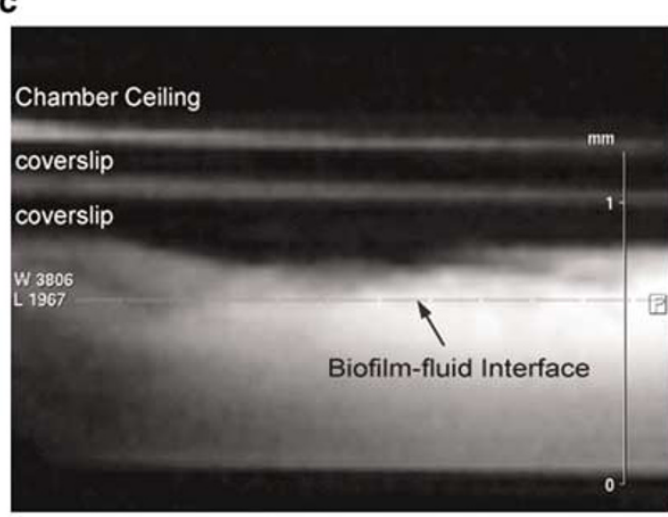

d

e

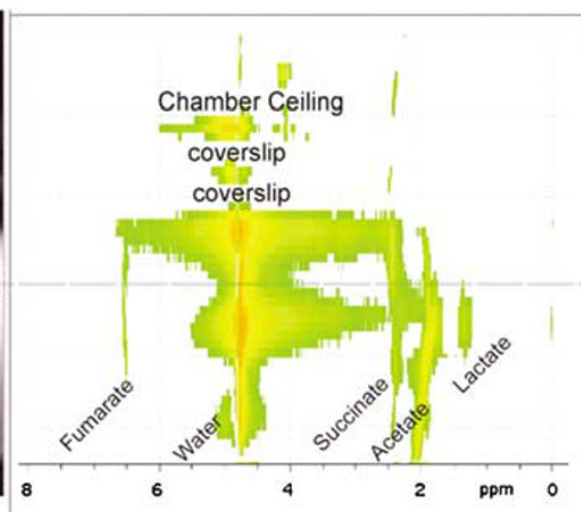

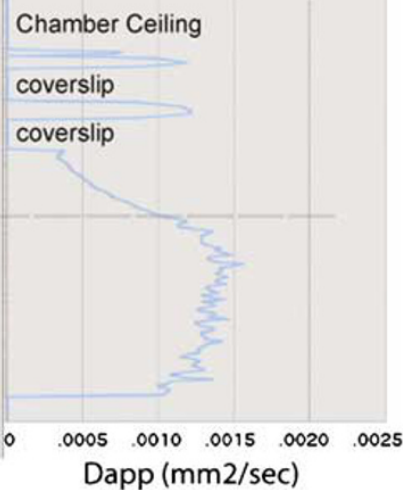

Figure 4 Correlated, depth-resolved respiratory-metabolism and diffusion-rate (water) measurements for a $S$. oneidensis biofilm fed lactate as a donor and fumarate as an electron acceptor. (a) Illustration showing localized spectral measurement volumes and locations for the depth-resolved metabolism measurement. Chemical spectra are averaged across a $2.5 \mathrm{~mm} \times 2.5 \mathrm{~mm} \times 31.2 \mu \mathrm{m}$ voxel and each voxel is an effective volume of $195 \mathrm{nl}$. (b) Biofilm-selective 3D MRI anatomic z-profile image of the biomass (gold color). (c) Waterselective MRI side profile showing two coverslips with water trapped between and a biofilm on the surface of the bottom coverslip. The arrow indicates the approximate biofilm-bulk fluid interface. (d) Depth-resolved MRSI data represented visually by a pseudocolor concentration map (red is high, green is low) showing relative metabolite concentration by color. The vertical direction of the metabolite map corresponds with spatial depth, and the horizontal direction is the spectral dimension. The resonance lines for lactate (1.33 p.p.m.), acetate (1.9 p.p.m.), succinate (2.4 p.p.m.) and fumarate (6.5 p.p.m.) and the broad water signature (4-5 p.p.m.) are shown. The vertical height is registered with the anatomical image in panel c and diffusion measurement in panel 4e. (e) Depth-resolved apparent water diffusion rate $\left(D_{\mathrm{app}}\right)$ profile collected for the same sample, showing that the diffusion rate drops linearly with biofilm depth starting near the location of the biofilm-bulk fluid interface.

approximate in vivo conditions (that is, an oral cavity). An MRI phase-encoding gradient was applied normal to the biofilm depth as contiguous thin volumes with $22 \mu \mathrm{m}$ step sizes corresponding to a volume of $140 \mathrm{nl}$ (see schematic in Figure 5a). The hydroxyapaptite disc was NMR-transparent and did not produce imaging contrast under the collection parameters that favored biofilm contrast. In this figure (Figures $5 \mathrm{~b}$ and $\mathrm{d}$ ), multiple views of the biofilm biomass on the hydroxyapatite surface (depicted in blue) are shown. A localized MRSI containing depth-resolved spectral (metabolic) data is shown in Figure 5d. Each of the depth (height registered) measurements for each $140 \mathrm{nl}$ voxel can be extracted and concentration profiles can be plotted. Here, we can resolve the changing sucrose (peaks located at 2.75-3.75 p.p.m.) concentrations at depths of 120, 60 and $20 \mu \mathrm{m}$ (top of the chamber, within the biofilm and near the hydroxyapatite surface, respectively) (Figure 5e). A spectral signature, characteristic of anomeric glucans (5.255.5 p.p.m.), is also detected. These glucans are a known virulence factor produced as a result of the action of glucosyltransferase on sucrose. In summary, we were able to image the developing biofilm on the hydroxyapatite and collect spectra at several depths from the surface.

\section{Discussion}

We have introduced the novel aspects of a noninvasive high-resolution spectroscopic/microscopic imaging capability. In particular, this system is employed to image and assess spatial distributions and fluctuations of metabolites in 3D communities of bacterial cells attached to surfaces (biofilms). Based upon combined optical laser scanning confocal and NMR microscopy, it provides biomass volume and distributions as well as time- and depthresolved metabolite concentrations in active biofilms for sample volumes on the order of $1 \mathrm{~mm}^{3}$. Previous researchers generally employ a standard NMR radial tube geometry with biofilm covering the circumference to study flow and diffusion characteristics of live biofilms (As and Lens, 2001; Seymour et al., 2004; Gjersing et al., 2005). What makes the current application very unique is the use of a planar perfusion chamber and the application of small-volume spectroscopy techniques to quantify 


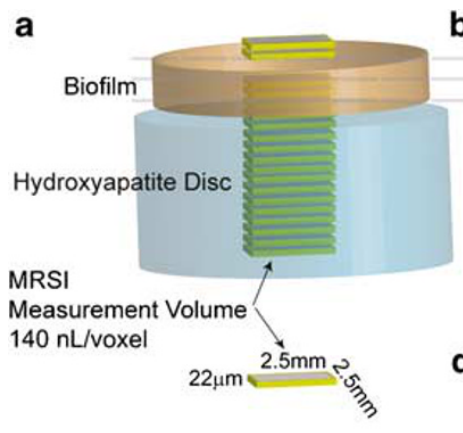

b

C

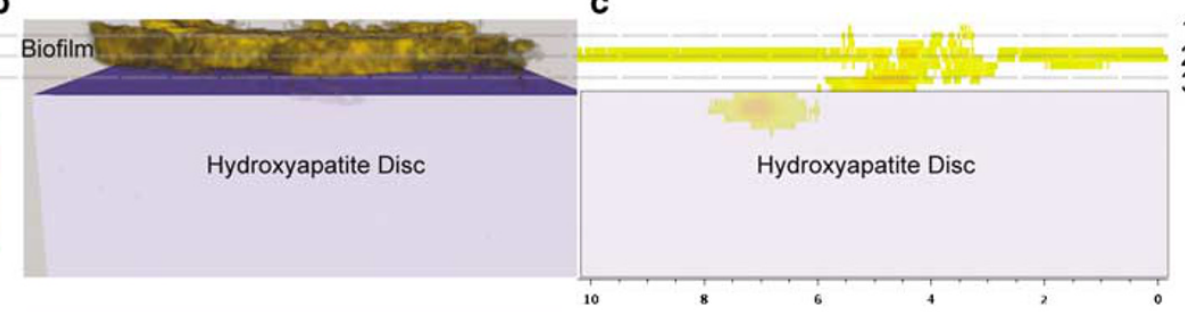

d

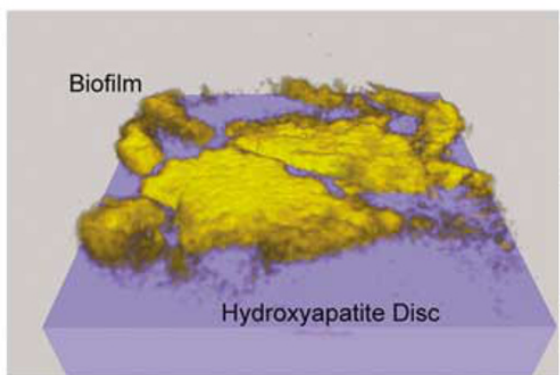

e

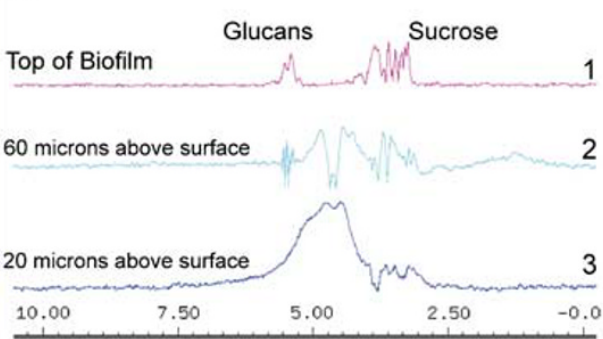

Figure 5 Imaging and depth-resolved metabolism measurements demonstrated on a biofilm of $S$. mutans grown on hydroxyapatite in a simple model of an oral cavity. (a) Schematic of localized spectra that were collected with a spectroscopic imaging (MRSI) single-spinecho sequence over a 2-mm-thick plane aligned orthogonal with the coverslip to minimize magnetic susceptibility distortions. Chemical spectra are averaged across a $2.5 \mathrm{~mm} \times 2.5 \mathrm{~mm} \times 22 \mu \mathrm{m}$ voxel and each voxel is an effective volume of $140 \mathrm{nl}$. (b) Depth-correlated biofilmselective 3D MRI anatomic z-profile image of $S$. mutans strain UA159 biofilm on the hydroxyapaptite disc (depicted in blue) rendered using Volocity software. The hydroxyapaptite disc was not visualized with the settings that provide contrast for the biomass and was effectively invisible. (c) Depth-resolved MRSI data represented visually by a pseudocolor concentration map (red is high, yellow is low) showing relative metabolite concentration by color. The vertical direction of the metabolite map corresponds with spatial depth and the horizontal direction is the spectral dimension, which is depth registered with the anatomical image in panel b. (d) Topdown 3D MRI view of the biofilm (gold color). (e) Each of the depth (height registered) measurements for each $140 \mathrm{nl}$ voxel can be extracted and concentration profiles can be plotted. Depths of 120,60 and $20 \mu \mathrm{m}$ (top of the chamber, within the biofilm and near the hydroxyapatite surface, respectively) are shown. Sucrose (peaks located at 2.75-3.75 p.p.m.) and a spectral signature characteristic of anomeric glucans (5.25-5.5 p.p.m.) were detected.

multiple metabolites in a temporal and depthresolved fashion. In addition, the available imaging MRI and CLSM, which can be employed on the same sample, provides complementary structural information. In addition to the gene expression information and the enhanced biofilm structure detail captured using fluorescence, CLSM can provide depth-dependent viability of cells using fluorescent stains or depth-dependent population distributions in a mixed-species biofilm by employing multi-color antibodies or fluorescent reporter molecules. ${ }^{1} \mathrm{H}$ NMR imaging effectively has no depth limitation and can be used to non-invasively monitor biomass distribution and volume over time. A number of MRI-sensitive targeted contrast agents including dual probes, such as magnetofluorescent nanoparticles for MRI and optical fluorescence, are being used in biological applications (Lee et al., 2006) and the further development of these for prokaryotic systems is likely.

In summary, in vivo MRI and MRS methods combined with CLSM offer many possibilities for investigating biofilm physiology and function. These combined techniques offer unprecedented structural, metabolic and transport information in near-real-time in a sealed flow chamber that are not achievable by other technologies. Together with a specialized chamber design, the system provides a controlled environment for live biofilm measure- ments and approximately 1D sample geometry to facilitate rapid spatially resolved depth and diffusion measurements. For example, linking ${ }^{1} \mathrm{H}$ NMRselective imaging, diffusion and spectroscopy defines the structure and boundaries of the biomass and enables interpretation of the metabolite depth profiles by location. These data are ideal for advanced biofilm modeling (Beyenal and Lewandowski, 2005), as they provide compatible, detailed experimental data for model validation. NMR methods are comparatively less sensitive than other spectroscopy methods because of the low (RF) transition energies involved. Nevertheless, we have shown that they can provide meaningful anatomic, metabolic and transport information on biofilms in a reasonable time and for physiologically relevant concentration ranges (cf. Figure 3c).

Future efforts to study 3D bacterial communities and more fully describe a biological sample of known or unknown species composition will benefit from this combined suite of imaging and spectroscopy techniques, which can be employed on a single sample. Investigation of the metabolic function in mixed microbial biofilms of environmental significance is one area where this technology can be applied. Prospective applications include monitoring rates of metabolism for multiple electron acceptor and donor pairs, substrate competition studies, metabolic shifts during perturbations 
and long-term effects of population changes on metabolite profiles, all of which can be conducted on relevant surfaces provided the surface does not induce significant NMR compatibility issues. Another key benefit of non-invasive and nondestructive techniques is the ability to retain the intact sample for other complementary invasive or destructive analyses to gain further insight into microbial interactions within these complex communities. Further work is planned to investigate the spatial profiles of acidic organic acids within mixedspecies oral biofilms on enamel surfaces to enhance our understanding of the demineralization process that occurs in oral caries disease. This technology is also readily applicable to other medically relevant biofilm systems, providing key metabolic information such as antibiotic/antimicrobial effects on metabolism and resurgence of metabolism after antibiotic treatment in a flowing system, which could be key information for battling many bacterial diseases.

\section{Acknowledgements}

We thank Dr JW Costerton (USC) for his advice and support as well as Dr Wenyuan Shi (UCLA) for the guidance and the use of $S$. mutans strain UA159. This research was supported by the Laboratory Directed Research and Development Program at the Pacific Northwest National Laboratory, a multiprogram national laboratory operated by Battelle for the U.S. Department of Energy under Contract DE-AC05-76RL01830. The research was performed in the Environmental Molecular Sciences Laboratory (a national scientific user facility sponsored by the Department of Energy's Office of Biological and Environmental Research) located at Pacific Northwest National Laboratory and operated for DOE by Battelle. Controlled cultivation of biofilms was performed in the Microbial Cell Dynamics Laboratory at PNNL.

\section{References}

Ajdic D, McShan WM, McLaughlin RE, Savic G, Chang J, Carson MB et al. (2002). Genome sequence of Streptococcus mutans UA159, a cariogenic dental pathogen. Proc Natl Acad Sci USA 99: 14434-14439.

As HV, Lens P. (2001). Use of $1 \mathrm{H}$ NMR to study transport processes in porous biosystems. J Ind Microbiol Biotechnol 26: 43-52.

Beyenal H, Lewandowski Z. (2005). Modeling mass transport and microbial activity in stratified biofilms. Chem Eng Sci 60: 4337-4348.

Brown TR, Kincaid BM, Ugurbil K. (1982). NMR chemical shift imaging in three dimensions. Proc Natl Acad Sci USA 79: 3523-3526.

Callaghan PT. (1984). Pulsed field gradient nuclear magnetic-resonance as a probe of molecular-organization. Proc Aust Biochem Soci 16: S7.

Costerton JW, Stewart PS, Greenberg EP. (1999). Bacterial biofilms: a common cause of persistent infections. Science 284: 1318-1322.
Davies D. (2003). Understanding biofilm resistance to antibacterial agents. Nat Rev Drug Discov 2: 114-122.

Dibdin G, Wimpenny J. (1999). Steady-state biofilm: practical and theoretical models. Methods Enzymol 310: 296-322.

Gjersing EL, Codd SL, Seymour JD, Stewart PS. (2005). Magnetic resonance microscopy analysis of advective transport in a biofilm reactor. Biotechnol Bioeng 89: 822-834.

Gonzalez-Gil G, Amonette JE, Romine MF, Gorby YA, Geesey GG. (2005). Bioreduction of natural specular hematite under flow conditions. Geochim Cosmochim Acta 69: 1145-1155.

Heidelberg JF, Paulsen IT, Nelson KE, Gaidos EJ, Nelson WC, Read TD et al. (2002). Genome sequence of the dissimilatory metal ion-reducing bacterium Shewanella oneidensis. Nat Biotechnol 20: 1118-1123.

$\mathrm{Hu}$ J, Wind RA, Mclean JS, Gorby YA, Resch CT, Fredrickson JK. (2004). High resolution 1H NMR spectroscopy of metabolically active microorganisms using non-destructive magic angle spinning. Spectroscopy 19: 98-103.

Junter GA, Coquet L, Vilain S, Jouenne T. (2002). Immobilized-cell physiology: current data and the potentialities of proteomics. Enzyme Microbial Technol 31: 201-212.

Lebihan D. (1990). Magnetic-resonance-imaging of perfusion. Magnetic Resonance in Medicine 14: 283-292.

Lee JH, Jun YW, Yeon SI, Shin JS, Cheon J. (2006). Dualmode nanoparticle probes for high-performance magnetic resonance and fluorescence imaging of neuroblastoma. Angew Chem Int Ed Engl 45: 8160-8162.

Lewandowski Z, Altobelli SA, Fukushima E. (1993). Nmr and microelectrode studies of hydrodynamics and kinetics in biofilms. Biotechnology Progress 9: 40-45.

Lewandowski Z, Altobelli SA, Majors PD, Fukushima E. (1992). Nmr imaging of hydrodynamics near microbially colonized surfaces. Water Science and Technology 26: 577-584.

Lewandowski Z, Stoodley P, Altobelli S. (1995). Experimental and conceptual studies on mass-transport in biofilms. Water Science and Technology 31: 153-162.

Majors PD, McLean JS, Fredrickson JK, Wind RA. (2005b). NMR methods for in-situ biofilm metabolism studies: spatial and temporal resolved measurements. Water Science \& Technology 52: 7-12.

Majors PD, McLean JS, Pinchuk GE, Fredrickson JK, Gorby YA, Minard KR et al. (2005a). NMR methods for in situ biofilm metabolism studies. J Microbiol Methods 62: 337-344.

Marsh PD. (2003). Are dental diseases examples of ecological catastrophes? Microbiology 149: 279-294.

Matthysse AG, Stretton S, Dandie C, McClure NC, Goodman AE. (1996). Construction of GFP vectors for use in Gram-negative bacteria other than Escherichia coli. FEMS Microbiol Lett 145: 87-94.

Mayer C, Lattner D, Schurks N. (2001). 13C nuclear magnetic resonance studies on selectively labeled bacterial biofilms. J Ind Microbiol Biotechnol 26: 62-69.

Minard KR, Holtom GR, Kathmann LE, Majors PD, Thrall BD, Wind RA. (2004). Simultaneous 1H PFG-NMR and confocal microscopy of monolayer cell cultures: effects of apoptosis and necrosis on water diffusion and compartmentalization. Magn Reson Med 52: 495-505.

Myers CR, Nealson KH. (1988). Bacterial manganese reduction and growth with manganese oxide as the sole electron acceptor. Science 240: 1319-1321. 
Parolin C, Montecucco A, Ciarrocchi G, Pedrali-Noy G, Valisena S, Palumbo $M$ et al. (1990). The effect of the minor groove binding agent DAPI (4,6-diamidino-2phenyl-indole) on DNA-directed enzymes: an attempt to explain inhibition of plasmid expression in Escherichia coli [corrected]. FEMS Microbiol Lett 56: 341-346.

Poole DF, Newman HN. (1971). Dental plaque and oral health. Nature 234: 329-331.

Revsbech NP. (2005). Analysis of microbial communities with electrochemical microsensors and microscale biosensors. Environmental Microbiology 397: 147-166.

Seymour JD, Codd SL, Gjersing EL, Stewart PS. (2004). Magnetic resonance microscopy of biofilm structure and impact on transport in a capillary bioreactor. J Magn Reson 167: 322-327.

Shanks JV. (2001). In situ NMR systems. Curr Issues Mol Biol 3: 15-26.

Stewart PS. (2001). Multicellular resistance: biofilms. Trends Microbiol 9: 204.

Stewart PS. (2003). Diffusion in biofilms. J Bacteriol 185: 1485-1491.

Stoodley P, Sauer K, Davies DG, Costerton JW. (2002). Biofilms as complex differentiated communities. Annual Review of Microbiology 56: 187-209.
Ullrich S, Karrasch B, Hoppe H, Jeskulke K, Mehrens M. (1996). Toxic effects on bacterial metabolism of the redox dye 5-cyano-2,3-ditolyl tetrazolium chloride. Appl Environ Microbiol 62: 4587-4593.

Van As H, Lens P. (2001). Use of (1)H NMR to study transport processes in porous biosystems. I Ind Microbiol Biotechnol 26: 43-52.

Vogt M, Flemming HC, Veeman WS. (2000). Diffusion in Pseudomonas aeruginosa biofilms: a pulsed field gradient NMR study. J Biotechnol 77: 137-146.

Vroom JM, De Grauw KJ, Gerritsen HC, Bradshaw DJ, Marsh PD, Watson GK et al. (1999). Depth penetration and detection of $\mathrm{pH}$ gradients in biofilms by two-photon excitation microscopy. Appl Environ Microbiol 65: 3502-3511.

Wimpenny JW. (1985). Novel growth systems. Microbiol Sci 2: 53-60.

Wind RA, Minard KR, Holtom GR, Majors PD, Ackerman EJ, Colson SD et al. (2000). An integrated confocal and magnetic resonance microscope for cellular research. J Magn Reson 147: 371-377.

Zachara JM, Fredrickson JK, Li SM, Kennedy DW, Smith SC, Gassman PL. (1998). Bacterial reduction of crystalline $\mathrm{Fe}^{+}$oxides in single phase suspensions and subsurface materials. American Mineralogist 83: 1426-1443.

Supplementary Information accompanies the paper on The ISME Journal website (http://www.nature.com/ismej) 